\title{
A Brief Talk on the 3D Scanning Reconstruction Program Based on Kinect and its Application
}

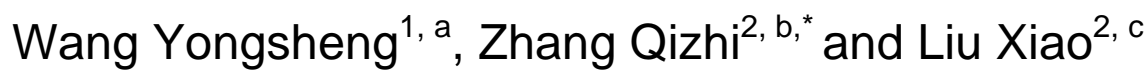 \\ ${ }^{1}$ School of art and design, Lanzhou Jiaotong University, Lanzhou 730070, China \\ ${ }^{2}$ School of art and design, Lanzhou Jiaotong University, Lanzhou 730070, China \\ a wys_Iz@126.com, ${ }^{\mathrm{b}}$ zhangqizhi1990@126.com, ${ }^{\mathrm{c}}$ cfeiio@sina.com
}

\begin{abstract}
By combining the Kinect (a somatosensory device) with a supporter and a rotary wheel and using a 3D scanning software named KScan3D to realize a quick scan of the human body, this paper constructs two scanning systems, one of which is equipped with a handheld single-camera comparing to the other adopting multiple fixed cameras, so as to generate virtual 3D models and provide various applications, such as printing 3D objects, generating web pages or making animations and virtual exhibition models.
\end{abstract}

Keywords: 3D scanning restructure; painted sculptures of Dunhuang; digitalization.

\section{Introduction}

The rapid growth of computer sciences and digital VR techniques has made it possible for using 3D scanning and printing technologies to realize digital 3D visual reconstruction. Such advancement has bee promptly applied to various areas including construction, medical care, archeology and industrial use. Compared to traditional modeling methods, 3D scanning and printing technologies are faster and more precise. Due to a wide range of utilities, human body modeling has become a desperately urgent need.

\section{3D Scanning Reconstruction}

As described during the experiment in this paper, data are first obtained through Kinect, a somatosensory camera sensor. Subsequently, the data are converted to a 3D mesh by using a software called Kscan3D. A mesh with 360 degree coverage can be achieved based on data showing different angles with the help of Kscan3D, which is capable of automatically capture and organize the 3D grip. Deleting unnecessary point from the data can smooth the data before the final combining and compositing to generate a complete model.

\subsection{Hardware Construction}

\subsubsection{Hardware Construction}

This system is designed to scan people standing about 1.6 to 1.9 meters tall, which means the supporter needs to be 2.5 meters high at the same level with the Kinect. Each Kinect is set about $64 \mathrm{~cm}$ away from the other and is responsible for scanning a part of the body. Then it is the software's job to connect the separated scanning results. The Kinect on the highest position scans the head and shoulders, while the second is in charge of the arms. Similarly, the third shoots the waist and the forth scans the legs and the feet. If this person is short, 3 Kinects may be enough, using the first Kinect for scanning the head and shoulders, the second for arms and waist, and the third for legs and feet. This experiment is conducted by using 3 Kinects. Besides, a rotary wheel running at a uniform velocity is required and its loading capacity has to be adequate to supporting a person for full 360 degree scanning. The rotary wheel is $66 \mathrm{~cm}$ away from the aluminum supporter as shown in Figure 1. 


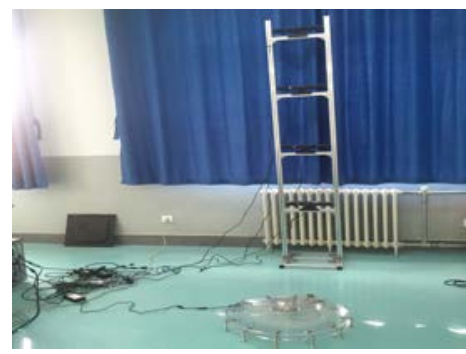

Fig. 1

\subsubsection{What is Kinect and how does it work:}

Kinect is a somatosensory camera sensor, providing multiple features, for instance, motion capture, image recognition, microphone input and voice recognition. Players are enabled to interact with virtual objects and other players in game as well as to share pictures and information with other $\mathrm{XBOX}$ users via internet. Kinect is first designed as a peripheral input equipment for the Xbox and later on Kinect for windows is developed for connecting with PC.

How does Kinect work: Kinect is a combination of various sensors, which comprises a color camera, a pair of depth cameras (one is used to emit infrared ray, the other is to received the returned ray), an array of microphones and a motor base, Kinect is able to work within a range from $1.2 \mathrm{~m}$ to $3.5 \mathrm{~m}$. As of Kinect for Windows, the range of the camera is shortened to $40 \mathrm{~cm}$ to $3 \mathrm{~m}$.

The depth camera uses IR to obtain the depth value of each point corresponding to the image returned (the depth value is actually the distance from the object to the Kinect in $\mathrm{mm}$ with $5 \mathrm{~mm}$ tolerance). The MIC array is actually a microphone arranged horizontally. Due to the gap, it can be used to determine where the speaker is and can reduce noises.

First of all, the program has to detect the connected Kinect for initialization. Second, the program is required to configure and enable its desired data flow, i.e. the expected data, including color information, depth data, bone data flow and IR data stream. Next, the corresponding data flow is obtained in accordance with the previous setup. Finally, it is to stop the Kinect and release PC resources.

\subsubsection{Precautions about the scanned object}

On selecting an object for scanning, it is the first thing to do to think over its features, such as its size, shape, weight and surface material. Sensors of Kscan3D and Kinect/Xtion are capable of scanning a lot of objects, for example, human body, furniture, house or even bigger stuff. Due to the resolution limit, distance from the senor to the object is not supposed to be less than $40 \mathrm{~cm}$ and the scanned object not smaller than a grown man's shoe. Objects that are too thin may not have satisfactory scanning results. Generally speaking, the best object to be scanned should be in neutral colors with matte opaque materials. It is hard to scan things of surface materials that are whether dark, reflective, transparent or translucent. Therefore, preparations should be made before scanning.

Environmental factors, including air, lights and movements, determine the success or failure of the experiment to a large extent. As of a physical object, it's a must to take its size into consideration and find a way to cover all the angles. For a small and light object, object is placed on the rotary wheel for scanning, while sensors are fixed. In general, the ambient light should cover all directions. As a matter of fact, sunlight may affect the working of IR sensors. It is not easy for scanning under sunlight. Therefore, if necessary, the scanning work is suggested to be done outside in a cloudy weather or be accomplished inside. During the scanning process, it is important to ensure the person or object motionless because movements may lead to a data acquisition failure.

\subsection{Software Introduction}

KScan3D is a 3D scanning software utilizing Kinect or Xtion to acquire point cloud data in a real-time manner, which allows multiple depth cameras to work simultaneously for scanning real objects and supports quick generating of complete 3D models. 


\subsection{Integration Solution to the 3D Scanning System}

There are mainly two types of 3D scanning solutions. One is a handheld scanning system with a single camera, while the other adopts multiple fixed cameras.

2.3.1 Handheld single-camera scanning system

Scanning modes for the handheld single-camera scanning system: the single-camera system has two scanning modes, which are individual scanning and batch scanning.

(1)Make sure the box of "enable batch scanning" within the scanning pane is not checked;

(2)Set up an initial delay (seconds);

(3)Turn the sensors towards the object and ensure the object can be seen from the real-time video feedback;

(4)Click the SCAN button

Figure 2 shows the scanning results.

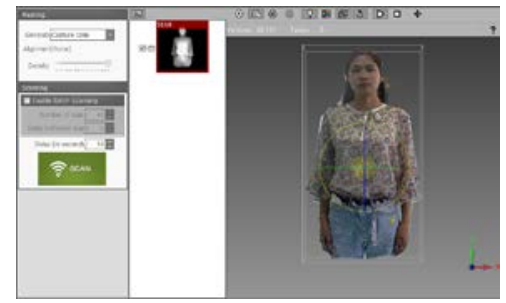

Fig. 2

Batch scanning:

(1)Check the box of "enable batch scanning" within the scanning pane;

(2)Select the number of objects to be scanned

(3)Set up a time delay between two scans

(4)Set up an initial delay (seconds);

(5)Turn the sensors towards the object and ensure the object can be seen from the real-time video feedback;

(6)Click the SCAN button

Figure 3 shows the scanning results.

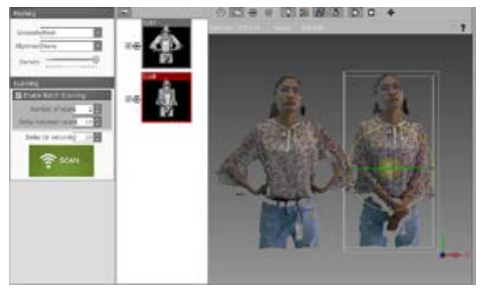

Fig. 3

2.3.2Fixed multiple-cameras system

1. Start the KScan3D software and make sure all sensor are working, as shown in Figure 4.

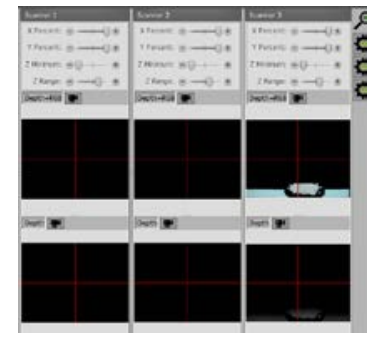

Fig. 4

2.Adjust the position of the sensor

If needed, it is Ok to move up or move down the position of the sensor in the list until the live response sequence complies with the sensor's actual position, as shown in Figure 5. 


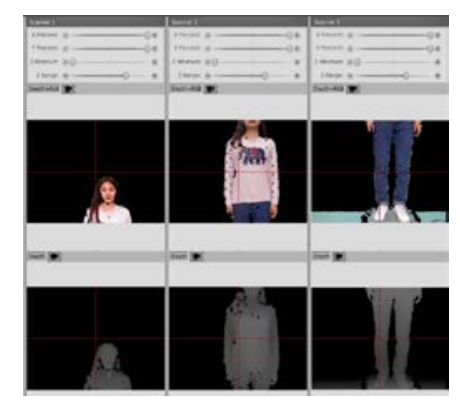

Fig. 5

3. Capture an individual scan.

Correctly set up the scanning options without checking the option for batch scanning. Adjust the parts to be scanned to the corresponding position and delete until the end, as shown in Figure 6 and Figure 7.

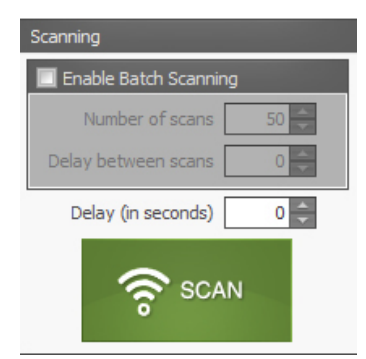

Fig.6

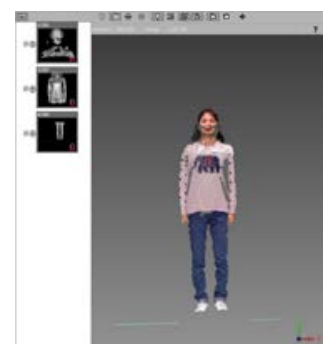

Fig.7

4. Capture a full-body scan

(1) Capture a full-body scan. Set up to the Capture Only mode and check the box of batch scanning with the number of scans set to 40 . The person should stand in the middle of the rotary wheel. Start the wheel before clicking the scan button as shown in Figure 8 and Figure 9.

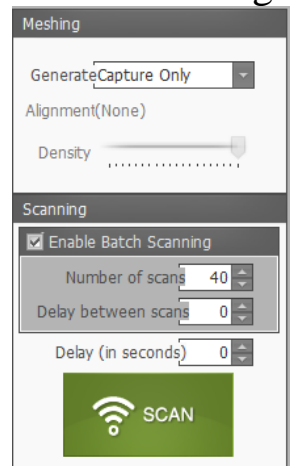

Fig. 8 


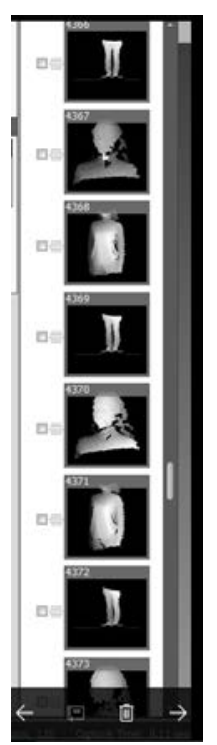

Fig.9

(2) Click the mesh-editing button and select the first picture. Press ctrl+a to select all thumbnails. Click the Point Cloud panel to set up the alignment as shown in Figure 10.

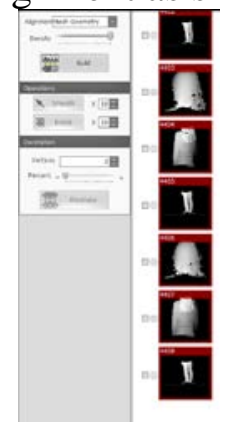

Fig.10

(3) Click the Build (for compositing) button and Kscan3D can turn each point into a mesh. In the end, delete unnecessary data. Using the basic mesh editing functions can help complete and export a high-quality result.

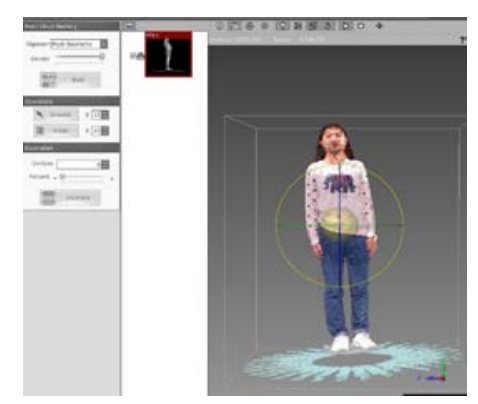

Fig.11

(4)Click the Finalize (finish) button to get the final model. Select the Finalize button for a combined mesh. Combination is required to be completed before export. The three properties in the finish step are used to adjust parameters like mesh density, inner fill and decimation, as shown in Figure 12. 


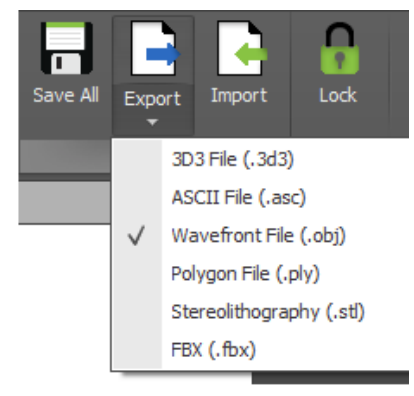

Fig.12

2.4 As long as the model is generated, select the Export button to export the mesh or point cloud to the current file. KScan3D can export the following file formats: fbx, obj, ply, stl, asc and 3d3.

(1) Select a format as shown in Figure 13

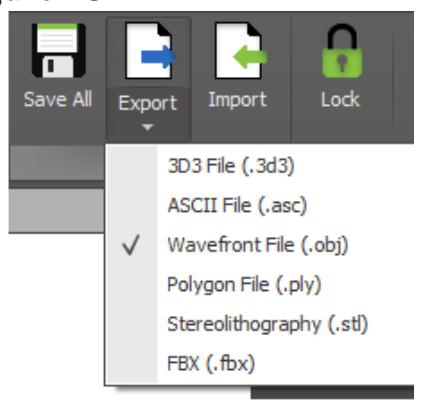

Figure 13

(2) Export data

In the control panel, load the mesh or point cloud to be exported. Please note that combine mesh is not supported for export. Click the Export button on the tool bar and an interface of folder selection will pop up as shown in Figure 14.

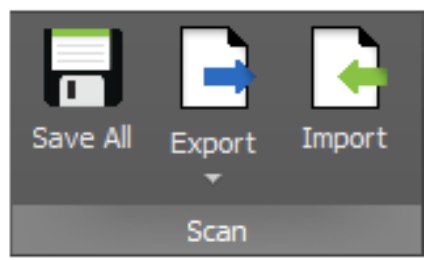

Fig. 14

Select a specified folder or create a new folder to store the mesh or pint cloud as shown in Figure 15.

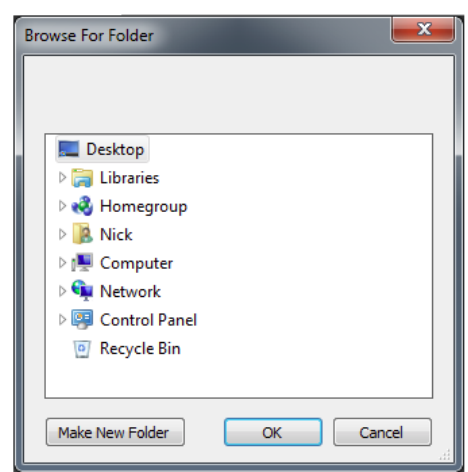

Fig. 15 


\section{3D printouts}

Technical support: 3D printing is a form of fast manufacturing technology. By "slicing" the drawing of the object to print into numerous layers, the processor heats up and presses the filament PLA (a new type of biodegradable material as the raw material) by utilizing the fuses deposition molding technology (FDM) to process each layer under computer control so as to get the formed 3D object. This is the most commonly adopted 3D printing method featuring high technical maturity and low cost.

Working steps of a 3D printer: use Kinect to scan the model and set up the printing parameters with the built-in software. Import the stl file from the memory card to the printer for printing. This paper uses a 3DA8 industrial printer manufactured by JGAURORA, which is able to print a maximum size of $350 * 250 * 300 \mathrm{~mm}$. The printing of large objects can be done through splicing. Coloring can be completed through spraying or manual painting.

Models created during this experiment are shown in Figure 16, Figure 17and Figure 18.

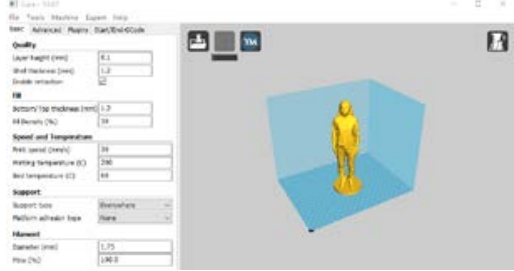

Fig.16

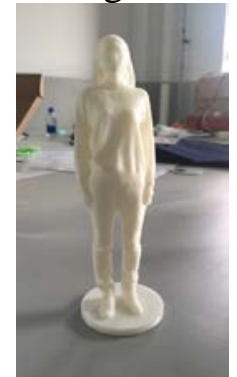

Fig.17

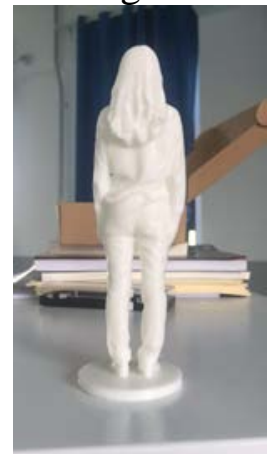

Fig.18

\section{Online interaction demo}

Blender is a virtualization engine for fast modeling, which is often used by modeling for games. As an open-source WebGL framework, Blend4Web can be used to create interactive webpages. Without going out, a user can have the chance to view 3D human models on webpages, check for details by rotating angles or zooming and share it to other users. This is impossible to realize by traditional browsing methods.

Through the Blender4web plugin, the scanned and finished human body model is allowed to be directly exported to a .html file. The Blender4web export mode is not included in the Blender so we have to manually install it: first, download the add-on corresponding to the Blender version from the official website for bledner4web. Then, in the Blender, click File->User Preferences->File-> scrips-> 
the zip containing the bledner4web add-on-> ->Save User Settings. Next, exit the blend and open File->User Preferences->Addons->import-export->Blend4web. After that, the .html option can be seen in the File->Export. Figure 19shows an exported webpage.

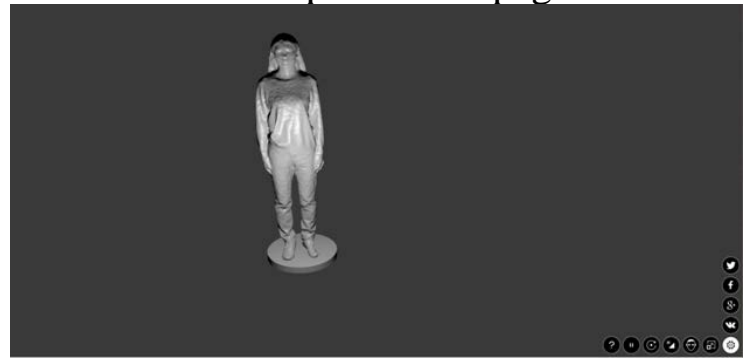

Fig.19

\section{Applied to animation and virtual exhibition}

Using the 3D scanning technology to conduct a comprehensive human body scan, a 3D model of human body and materials can be obtained. With parameter adjustment to enrich the changes and combinations, once these color models are inputted into 3D animation software like 3dsmax, fast, accurate and vivid performance can be achieved as well as virtual exhibition, which has greatly boosted the diversified development of the animation industry.

\section{Conclusion}

3D scanning and printing technologies have made it possible to express human models in a perfect, accurate and quick manner by inputting the scanned 3D information into a computer. This paper utilizes two scanning methods for human body modeling and studies their feasibility by printing a human model. However, due to scanning angles, the model may have flaws affecting the accuracy of modeling, which need to be improved in the future.

\section{References}

[1]System of Online Fitting Room Based on Web [J], Yang Wenwen, Guo Jianan and Yang Xiaodong, Computer Era, 2015, 5

[2]Application of 3D Scanning and Printing Technologies to the Repair of Crack Decorative Components [A], Jiang Yueju, Lv Haijun, Yang Xiaoyi, Xu Wei and Ma Xing Sheng, Construction Technology, 2016,12

[3]Research on the 3D Scanning and Printing of Human Head [A], Song Junfeng, Shenyang University of Technology, 2016

[4]Development of the 3D Printing Technology and its Software Implementation [J], Shi Yusheng, Zhang Chaobai, Bai Yu and Zhao Zuye, Science China Information, 2015

[5]Present Research on the 3D Printing Technology and Critical Know-Hows [J], Journal of Material Engineering, Zhang Xuejun, Tang Siyi, Zhao Hengyue, Guo Shaoging, Li Neng, Sun Bingbing and Chen Bingqing, 2016, 2

[6]Research on the Technology and Working Principle of Kinect [J], Shi Manyin, Natural science journal of Harbin Normal University, 2013, 6 